\title{
GTIP eHealth: regroupement de 100000 fournisseurs de prestations
}

\section{Alexander Zimmer}

Dr méd., membre du Comité central et responsable du département Numérisation/eHealth

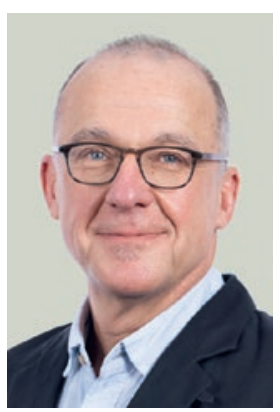

Alors qu'un événement providentiel bouleversait le monde il y a 2021 ans, la fin de l'année dernière a été le théâtre d'un événement qui mérite d'être mentionné: la création de l'association GTIP eHealth [1]. Il serait naturellement exagéré de voir un quelconque lien entre ces deux événements. Il n'en reste pas moins que le regroupement en une seule et même association de dix sociétés faîtières nationales, représentant au total 100000 professionnelles et professionnels de la santé, est un événement marquant.

GTIP eHealth est l'abréviation de groupe de travail interprofessionnel dans le domaine de la cybersanté. La FMH, pharmaSuisse, Physioswiss, l'Association suisse des infirmières et infirmiers (ASI), la Fédération suisse des psychologues (FSP), l'Association suisse des diététicien-ne-s (ASDD), l'Association suisse des ergothérapeutes (ASE), ChiroSuisse, la Fédération suisse des sages-femmes (FSSF) et la Société suisse des médecins-dentistes (SSO) se sont donné pour objectif d'optimiser l'échange de données informatisées entre les fournisseurs de prestations du domaine de la santé. Il est en effet essentiel de disposer d'un échange de données utile pour assurer le succès de la numérisation. Or, les attentes en termes de qualité d'un tel outil ne peuvent être définies que par les professionels de la santé eux-mêmes.

\section{Réussir la transition numérique dans le} domaine de la santé nécessite une participation active des fournisseurs de prestations.

L'association «GTIP eHealth» a été développée à partir $\mathrm{du}$ «Groupe de travail interprofessionnel pour le dossier électronique du patient» (GTIP DEP), fondé en 2014. L'objectif de départ des associations était de structurer de manière utile les informations dans le dossier électronique du patient (DEP). Par la suite, des recommandations ont été formulées pour les domaines de la cybermédication, du rapport de sortie électronique et des eAllergies et intolérances afin de soutenir eHealth
Suisse [2], ce qui a ensuite servi de base à la Confédération et aux cantons pour la mise en place d'un DEP adapté à la pratique. Il était alors clair pour le GTIP DEP que l'outil obtenu devait permettre une interopérabilité entre les différents systèmes informatiques devant communiquer avec le DEP.

Entre-temps, les conditions-cadre concernant le DEP ont cependant évolué. On s'est rendu compte que le DEP ne pourra satisfaire que de manière limitée aux

\section{Le GTIP eHealth prend son rôle à cœur pour} faire avancer avec succès la numérisation dans le domaine de la santé.

attentes des fournisseurs de prestations. Il ne prévoit en effet pour le moment aucune communication dirigée entre les professionnel-le-s du domaine de la santé, encore moins un soutien dans le processus d'échange de données au niveau interprofessionnel. Dans sa conception actuelle, un échange dynamique de données entre les fournisseurs de prestations et les patientes et patients n'est malheureusement pas possible. Ce dysfonctionnement doit être compensé par des services supplémentaires liés au DEP, par exemple par un service permettant un échange de données en dehors du DEP. C'est exactement sur cet objectif que le GTIP eHealth se concentre à présent. Pour réussir la transition numérique dans le domaine de la santé, une participation active des fournisseurs de prestations est nécessaire. Ce n'est qu'ensemble qu'ils pourront faire entendre leur voix auprès de la politique et de l'industrie. Le GTIP eHealth prend son rôle à cœur pour faire avancer avec succès la numérisation dans le domaine de la santé.

\section{Références}

1 GTIP eHealth. Fondation d'une communauté de travail interprofessionnelle en faveur d'une meilleure efficacité dans la numérisation du système de santé. Communiqué de presse du 3 novembre 2021. www.fmh.ch/fr/politique-medias/communiques-de-presse.cfm

2 Formats d'échange existants. ehealthsuisse. https://www.e-healthsuisse.ch/technik-semantik/semantische-interoperabilitaet/austauschformate.html 